DOI: 10.21892/978-958-5547-66-7.5

\title{
5. La responsabilidad médica y sus títulos de imputación a la luz de la jurisprudencia del Consejo de Estado: un análisis de 1991
}

\author{
Juan Felipe Solórzano Quintero²
}

\section{Introducción}

El presente escrito pretende mostrar de manera introductoria los títulos de imputación derivados de la actividad médica, en los casos que implique responsabilidad extracontractual del Estado.

Para la realización de este documento se tuvieron en cuenta las sentencias proferidas por el Consejo de Estado desde el año 1991, momento en que se expidió la Constitución, hasta el primer trimestre de 2017. El propósito del artículo se centra en poder evaluar si el Consejo de Estado ha tenido una línea jurisprudencial sólida y fundamentada en materia del acto médico.

Sin embargo, evidenciaremos que en los primeros 16 años el Consejo de Estado varió constantemente, situación que impedía tener claro el título de imputación que debía ser alegado por la parte demandante. Incluso, en la actualidad se encuentran fallos en los cuales la parte demandante alude aún al régimen de la falla presunta.

Esto es reflejo de las constantes oscilaciones del máximo tribunal contencioso administrativo en Colombia. Si bien, es cierto que en materia de responsabilidad médica desde el año 2006 se habla de la aplicación del régimen de la falla probada, denotaremos que los cambios del Consejo son

1 El escrito que se presenta es un aparte actualizado y mejorado del trabajo de tesis para optar por el título de Magister en Derecho administrativo por la Universidad del Rosario titulado "Elementos jurídicos para una política de prevención del daño antijurídico: consentimiento informado".

2 Abogado de la Universidad del Rosario. Magíster en Derecho Administrativo de la Universidad del Rosario. Doctor en Derecho de la Universidad de Salamanca, España. 
repentinos, por lo cual, no podremos estar seguros que será este el régimen aplicable por un largo período.

El trabajo presentado se desarrolla en tres etapas que pueden ser divididas de acuerdo a las transformaciones que el Consejo de Estado generó. Tenemos como primer periodo el comprendido entre los años 1991 a 2000. Posteriormente, hubo una transición en la cual se introdujo como sistema de aligeramiento probatoria la carga dinámica de la prueba, y finalmente desde el año 2006 a la actualidad existe propiamente un retorno al título de imputación primigenio, es decir, la falla probada.

1. Los títulos de imputación en materia de responsabilidad médica

El tema de responsabilidad extracontractual en Colombia generó controversia luego de la entrada en vigencia de la Constitución Política de 1991. El artículo 90 de la Carta estableció el principio general de la responsabilidad patrimonial del Estado y excluyó aparentemente el régimen de culpa. En la medida que solo se estableció el daño antijurídico y se consagró únicamente un régimen objetivo (Fernández Muñoz, 2008).

Al respecto, Fernández Muñoz (2008, p. 82)33 sostiene: "Según los defensores de esta doctrina, sólo el daño constituye la fuente de la obligación indemnizatoria, desplazándose el fundamento de la responsabilidad administrativa, del concepto subjetivo de la antijuridicidad de la conducta, al concepto objetivo de antijuridicidad del daño".

Así, parte de la doctrina reaccionó y arguyó que todos los actos que impliquen responsabilidad estatal no pueden encuadrarse únicamente dentro del régimen de responsabilidad objetiva. Si se aplica el régimen objetivo en todas las actividades desarrolladas por la Administración implicaría, por ejemplo, que en el ámbito de la responsabilidad médica

3 Serrano Escobar (2007) sostiene al respecto que "el Consejo de Estado, en una primera etapa, interpretó que la noción de daño antijurídico daba lugar a una responsabilidad objetiva, pero como no encontró la manera de aplicar tal criterio de imputación a la responsabilidad médica estatal, que para el máximo Tribunal de lo contencioso administrativo se debía seguir gobernando por criterios de imputación subjetivos, como la falla del servicio, utilizó la tradicional clasificación de la obligaciones de medio y resultado, sosteniendo que esta noción de daño antijurídico no se aplica a la obligación de medio, en la cual se ha catalogado tradicionalmente a la medicina, para seguir radicando la responsabilidad médica en criterios de imputación subjetivos, atenuándolo con la noción de falla del servicio presunta". En el mismo sentido véase Sarmiento García (2001 p.47-48). 
todas las intervenciones que reporten un perjuicio para el paciente tendrían que indemnizarse.

De esta forma, se eliminaría el concepto de obligación de medios que tiene el profesional de la salud. De manera concomitante y al no contarse con la falla del servicio como elemento constitutivo de responsabilidad el fisco sufriría grandes perjuicios, ya que únicamente la Administración podría exonerarse de culpa demostrando la causa extraña. Por lo anterior, se consideró que la intención del constituyente fue la de darle rango constitucional a la cláusula de responsabilidad del Estado.

En consecuencia, el Consejo de Estado hizo una clasificación que suplió las necesidades actuales de la responsabilidad extracontractual del Estado. Según Fernández Muñoz (2008).Dicho tribunal sostuvo:

Por un lado, se hablará de daño antijurídico subjetivo, al involucrar la noción de falla del servicio (antijuridicidad de la conducta de la administración) y donde el daño resulta antijurídico en la medida que el particular no está obligado a soportar un daño antijurídico objetivo, equivalente a los eventos de responsabilidad objetiva(antijuridicidad del daño), cuando el daño resulta causado no obstante la administración haya obrado adecuadamente (sin falla) y debe ser indemnizado por cuanto el particular no está obligado a soportarlo sólo en su patrimonio.

Ahora bien, el desarrollo que ha tenido la jurisdicción contencioso administrativa desde el año 1991 en relación con el tema de la responsabilidad médica, ha sido enfocado bajo la falla médica ${ }^{4}$ dentro del régimen subjetivo, cambiando de falla probada a presunta y usando por un lapso el régimen de cargas dinámicas de la prueba en su línea jurisprudencial.

Gil Botero (2011) manifiesta que el máximo tribunal contencioso administrativo en materia de títulos de imputación y del régimen

$4 \quad$ La falla del servicio incluye tres elementos los cuales son: "1) el daño antijurídico sufrido por el interesado, 2) la falla del servicio propiamente dicha, que consiste en el deficiente funcionamiento del servicio porque no funcionó cuando ha debido hacerlo o lo hizo de manera tardía o equivocada, y, finalmente. 3) Una relación de causalidad entre dos elementos, es decir, la comprobación de que el daño se produjo como consecuencia de la falla del servicio" (Ruíz Orejuela, 2006). 
probatorio en la responsabilidad médica no ha elaborado una línea jurisprudencial rigurosa. Para nosotros, como adelante lo demostraremos, el Consejo de Estado analiza sus providencias bajo la denominada figura de jurisprudencia indicativa ${ }^{5}$. Se trata de una situación que permite cambios jurisprudenciales repentinos, los cuales van en contravía de la seguridad jurídica que los poderes públicos deben garantizar.

Entrando en la materia debemos manifestar que la falla del servicio tiene por objeto imputar responsabilidad a la Administración cuando su actuar no es prudente o diligente y como resultado se genera un daño que la persona (administrado) no debe soportar (Consejo de Estado de Colombia, 1989). En este régimen la ausencia de culpa es un elemento primordial que exonera de responsabilidad. Así, encontramos que existe una diferencia con el régimen objetivo, por cuanto en este último la conducta no es determinante en la exoneración de responsabilidad. Igualmente, no es necesario individualizar el sujeto que causa el daño, basta con demostrar que la Administración fue la causante del perjuicio.

Pero como manifestamos anteriormente, el máximo tribunal contencioso administrativo ha utilizado como títulos de imputación la falla en el servicio probada o presunta, lo cual repercute directamente en los elementos probatorios que el demandante debe demostrar al momento de la demanda. De esta manera se pueden evidenciar tres grandes etapas en la línea seguida por el Consejo de Estado:

i) Desde el año 1990 al 2000, momento en el cual cambio el título de imputación de falla probada a presunta.

ii) En el año 2000 cuando se incorporó la carga dinámica de la prueba.

5 López Medina (2009) define este concepto como: "bajo esta última las citas a casos anteriores tienden a ignorar criterios de analogía fáctica y a concentrarse más bien en la definición de conceptos jurídicos hecha en las sentencias anteriores; en consecuencia, cada caso nuevo se decide de conformidad con la Ley y con el concepto jurídico anteriormente definido, con baja sensibilidad a la fuerza gravitacional de fallos anteriores análogos por su hechos y circunstancias; finalmente, esta concepción de la jurisprudencia permite cambios frecuentes de criterio sin que se defina o discuta la doctrina jurisprudencial fijada en casos análogos" ( p.109-110). 
iii) El retorno a la falla presunta a partir de 2000, pero reafirmado en 2006. Es menester mencionar que este régimen se encuentra vigente en la actualidad.

\section{Cambio de la falla probada a la falla presunta (1990-2000)}

En 1990, la falla probada era el título de imputación aplicable a la actividad médica. No obstante, la falla presunta empezó a insinuarse como obiter dictum ${ }^{6}$ a partir de la sentencia del 24 de octubre de 1990, exp 5902.

La mencionada providencia dio lugar a la formulación de ciertos postulados, a saber:

i) El actor a la hora de demandar debe probar las obligaciones y su incumplimiento sustentado en los artículos 1757 del Código Civil y 177 del Código de procedimiento Civil(Gil Botero, 2012).

ii) La Administración será la interesada en mostrar que su actuar fue el correcto (Consejo de Estado de Colombia, 1990).

iii) Basta probar el daño y que éste tuvo lugar por la falla de la Administración. Además manifestó el Consejo de Estado que "el art. 1604 debe aplicarse, como en varias oportunidades lo ha sostenido la Corte Suprema de Justicia, tanto en el campo de la responsabilidad contractual como extracontractual y si ello es así los eximentes de responsabilidad (fuerza mayor o caso fortuito) o la culpa exclusiva de la víctima serán de cargo del deudor (aquél a quien se imputa la responsabilidad)"(Consejo de Estado de Colombia, 1990).

Posteriormente, en la sentencia del 30 de julio de 1992, exp. 6897 se estableció con claridad que la falla presunta es aplicable a los temas de responsabilidad derivados del acto médico. Se consideró que el paciente no contaba con el suficiente conocimiento de las terapéuticas o procedimientos

6 Entiéndase como obiter dictum "La expresión designa todos aquellos pasajes de las sentencias en los que, por abundancia argumentativa propia del derecho jurisprudencial, se dicen las cosas "de pasada" o incidentalmente, sin que constituyan el meollo del asunto jurídico que se está resolviendo. Estos argumentos son, generalmente, superabundantes, eruditos y de mera referencia y no tiene relación directa con la parte dispositiva (decisum) de la sentencia. (Gil Botero, 2012, 490) 
que le son aplicados, razón por la cual el profesional de la salud o las instituciones médicas se encuentran en mejor posición de demostrar su actuar diligente y prudente (Consejo de Estado de Colombia, 1992b).

Los defensores de la falla presunta consideran que, al invertirse la carga de la prueba, el juez conocerá de primera mano el procedimiento aplicado. De esta manera se garantiza un juicio más justo. En este orden de ideas, al demandante solo necesita probar el daño y la relación de causalidad. En consecuencia, se sustrae de la prueba de la falla del servicio, que trae consigo explicaciones científicas, las cuales pueden resultar muy complejas para el paciente (Parra Guzmán, 2010). Asimismo, se dice que el demandado se puede liberar de responsabilidad demostrando diligencia y cuidado en su actividad (Tamayo Jaramillo, 2001).

Este cambio jurisprudencial se puede atribuir a que la anterior posición (falla probada) fue considerada "errónea, por ser contraria a los valores, objetivos, principios y derechos en los que se fundamenta el ordenamiento jurídico. (...) para evitar prolongar en el tiempo las injusticias del pasado, haciendo explícita tal decisión" (Corte Constitucional de Colombia, 2001). El Consejo de Estado quiso hacer ver que, en materia de responsabilidad médica, el demandado estaba en una mejor posición para probar que su conducta se adecuaba a los parámetros de la lex artis. Sin embargo, posteriormente volvió a hacer uso de la falla probada Respecto del cambio de títulos de imputación la doctrina estableció dos problemas. El primero de ellos fue la intermitencia o la no aplicación del régimen de falla presunta.

En opinión de Tamayo Jaramillo, la jurisprudencia no aplicó del todo la falla presunta, por cuanto en dichas sentencias se percibe una falla demostrada hasta la saciedad, en criterio de este autor, se siguió aplicando la teoría de la falla probada (Tamayo Jaramillo, 2001).

En este plano se observa lo siguiente: los fallos del Consejo de Estado del 30 de julio de 1992 y 13 de agosto de 1992, consideraron la falla presunta del servicio, no obstante, doctrinantes como Serrano Escobar estiman que se seguía aplicando el régimen de falla probada (Serrano Escobar, 2007). Esta afirmación fue apoyada por Herrera Ramírez, quien sostuvo que la falla probada fue usada hasta 1995.

Un segundo problema fue el desequilibrio en materia probatoria dentro del régimen de falla presunta, el cual puso en una situación de 
prevención al personal médico. Respecto este asunto, Parra Guzmán (2010) anotó:

Admitir una presunción de culpa en el campo de los errores profesionales médicos podría llevar a consecuencias desastrosas en cuanto que, si de un lado, se libera a la víctima de una difícil prueba muchas veces imposible de destruir: por mucho que valga el médico no se puede trabajar con la seguridad de una máquina y muchas veces aun estando seguro de haber actuado según los dictados de la ciencia, la naturaleza puede traicionar su esperanza sin su culpa.

En el mismo sentido, Tamayo Jaramillo se opone a la aplicación de la falla presunta, por considerar que en la praxis es muy difícil para el médico probar ausencia de culpa por la insuficiencia de medios probatorios y cree que al darle paso a la falla presunta se permitiría también la presunción del nexo de causalidad (Tamayo Jaramillo, 2001).

Se puede sustentar en la aleatoriedad de la actividad y por ende a su vez es de medios y se debe probar la culpa acudiendo en ocasiones a la culpa virtual que es una culpa probada a través de indicios. (...) En efecto, presumir la culpa del médico, sin saber cuál fue la causa del daño, conduce, nada más ni nada menos, a una presunción de causalidad que no es más que una responsabilidad objetiva. (p 57)

Vemos, entonces, cómo parte de la doctrina no se adhirió del todo a la posición establecida por el Consejo de Estado, pues consideraban que se dio pasó "de una posición rígida a favor de los médicos, para caer en otra igualmente rígida a favor de las víctimas" (Tamayo Jaramillo, 2001) y complementan su argumento diciendo que la falla probada es la que se debe aplicar. Paralelamente, Tamayo Jaramillo considera que se dio cabida a la presunción de la relación de causalidad lo cual hace más gravosa la posición del profesional de la salud. Así, en aras de alivianar la carga probatoria de los pacientes se perjudicó gravemente a los médicos. Es pertinente mencionar que en ocasiones el médico se encuentra únicamente con su paciente y por ello tendría como única prueba la narración de sus hechos, los cuales son fácilmente impugnables. Aunado a ello, la historia 
clínica podría ser objetada y por ende la presunción no podría desvirtuarse (Tamayo Jaramillo, 2001).

Siguiendo con el análisis jurisprudencial, la sentencia del 13 de agosto de 1992, exp. 7274 continuó aplicando la falla presunta (Consejo de Estado de Colombia, 1992). Esta vez para su aplicación se dio por la aplicación de las citas de referencia conceptual común (López Medina, 2009). El análisis judicial no parte de situaciones fácticas delimitadas, sino el juez se remite a conceptos jurídicos comunes abstractos. Para el caso el concepto utilizado fue el traslado de la prueba a cargo del médico o la entidad hospitalaria por contar con mayores facilidades en la prueba de un actuar diligente y prudente. En este entendido se tomó como base para fallar la sentencia del 30 de julio de $1992 \exp 6897$.

Posteriormente, mediante sentencia del 24 de agosto de 1992, exp. 6754, el Consejo de Estado reiteró el régimen de falla presunta, que se iría a aplicar hasta el año 2000. Vale decir, que en este fallo se expuso de manera diáfana que a la parte demandante le corresponde demostrar el daño y la relación de causalidad, lo único que se presume es la falla del servicio, en otras palabras, el actor no deberá demostrar la conducta tardía, omisiva o irregular de la administración debido a que se presume. En este sentido el Consejo de Estado de Colombia (1992) manifestó, que:

La exoneración de carga de la prueba que implica la noción de falla presunta es apenas relativa, porque al actor le incumbe en tales casos probar como mínimo los supuestos que permiten la operancia de la presunción. Así, en el caso de que alguien alegare que resultó lesionado por una intervención quirúrgica inadecuada, deberá probar, en términos generales, que se le prestó el servicio en tal fecha y que sufrió el daño cuya indemnización pretende.

Estamos de acuerdo con Enrique Botero Gil, al sostener que el Consejo de Estado no aplicó de manera rigurosa y bajo la misma estructura argumentativa la falla presunta. Sustenta que la Sección Tercera se circunscribió únicamente a exponer unas explicaciones a favor de la inversión de la prueba " $y$ en beneplácito de la parte demandante, a partir de comprobar que existe facilidad de prueba derivada de la experiencia, pero sin ninguna aproximación teórica normativa, salvo en la primera de las sentencias citadas, en la cual se definió 
que el artículo 1604 del Código Civil constituye el sustrato del morigeramiento probatorio mencionado" (Gil Botero, 2011. p 497).

Con lo anterior, podemos afirmar que el Consejo de Estado no formó una línea jurisprudencial consolidada en materia de responsabilidad médica (Consejo de Estado de Colombia, 2006a), solamente se limitó a resolver los casos basándose en unos conceptos dados con anterioridad, que no se relacionan directamente con la situación fáctica. Esta situación impide la formulación en una línea jurisprudencial sólida y en consecuencia de subreglas que permitan la solución de casos posteriores.

\section{La carga dinámica de la prueba}

Mediante providencia del 10 de febrero de 2000 exp. 11878 se adoptó el régimen de la carga dinámica de la prueba, como método de aligeramiento probatorio. Sin embargo, en este periodo el Consejo de Estado no del todo aplicó éste régimen (Consejo de Estado de Colombia, 2000). En el entendido que siempre el facultativo tuvo la carga de la presunción de falla y de la relación de causalidad. Por lo tanto, más que hablar de carga dinámica de la prueba se generó el afianzamiento de un régimen estático en materia probatoria.

Para Fernández Muñoz (2008):

Se estaba haciendo recaer toda la carga sobre el demandado, denotando como estática la carga el demandante y olvidando que al juez le correspondía esforzarse también por conocer la realidad de los eventos, utilizando los poderes que posee en materia de pruebas, todo con el fin de no perjudicar a la parte gravada con la inversión de la carga. (p.88)

A tal efecto, Tamayo Jaramillo (2001) manifiesta que el Consejo de Estado sustentó la presunción de la falla en el servicio, acudiendo a la carga dinámica de la prueba en donde el demandante y el demandado según el caso serían los encargados de allegar las pruebas. Concluye que el concepto de la carga dinámica no puede ser mirado desde un punto rígido, por cuanto en la praxis, siempre se le implicó soportar la carga al médico. Finalmente dice: 
(...) por ello consideramos que por carga dinámica de las pruebas ha de entenderse la obligación que tiene el médico de no permanecer pasivo a la espera de que se pruebe su culpabilidad, sino por el contrario, de colaborar con su propia versión y documentos al establecimiento de una verdad real, pero sin que ello implique una presunción de culpa en su contra, a menos que ésta aparezca expresamente consagrada por el legislador. (p. 94)

Aunado a lo anterior, la aplicación del sistema de las cargas dinámicas denota problemas prácticos, dado que el juez es el encargado de asignar la carga a las partes, y como es sabido en el proceso contencioso administrativo no existe una etapa idónea en la que se permita tal asignación (Gil Botero, 2011).

El Consejo de Estado posteriormente se percató de la dificultad práctica que tenía la aplicación del sistema de cargas dinámicas (Consejo de Estado de Colombia, 2006a). El repentino cambio da cuenta que el Consejo de Estado no realizó un análisis jurisprudencial riguroso. Por el contrario, muestra el uso de la denominada jurisprudencia indicativa, la cual "no tiene un peso suficiente como para crear cargas espaciales de argumentación al momento de cambio jurisprudencial” (López Medina, 2009).

\section{El retorno al sistema de falla probada}

Según Gil Botero (2011) a partir del año 2000, con la sentencia del 10 de febrero, se retornó al sistema de falla probada ${ }^{7}$, por cuanto el Consejo de Estado hizo énfasis en que el actor era quien debía asumir la carga de la prueba. Según el autor en cita la carga dinámica de la prueba no se aplicó en ningún caso en concreto, por parte del máximo tribunal contencioso administrativo.

Para el año 2006 se eliminó el sistema de falla presunta y carga dinámica de la prueba, para aplicar en estricto sentido la falla probada. Por lo tanto, le corresponde a la parte actora demostrar los elementos

7 Sobre la falla probada del servicio médico asistencial véase: (Consejo de Estado de Colombia, 2008b, 2008c, 2010b, 2010a, 2012). 
de responsabilidad, así sea de forma indiciaria (Consejo de Estado de Colombia, 2006b). Las razones que se tuvieron para el cambio de sistema a aplicar fueron:

La presunción de la falla del servicio margina del debate probatorio asuntos muy relevantes, como el de la distinción entre los hechos que pueden calificarse como omisiones, retardos o deficiencias y los que constituyen efectos de la misma enfermedad que sufra el paciente. La presunción traslada al Estado la carga de desvirtuar una presunción que falló, en una materia tan compleja, donde el alea constituye un factor inevitable y donde el paso del tiempo y las condiciones de masa (impersonales) en las que se presta el servicio en las instituciones públicas hacen muy compleja la demostración de todos los actos en los que éste se materializa (Consejo de Estado de Colombia, 2006).

Al respecto, el Consejo de Estado en auto del 22 de mayo de 2008, expediente No. 26427 expresó:

De manera reciente la sala ha recogido las tesis de la presunción de la falla médica, o de la distribución de las cargas probatorias de acuerdo con el juicio sobre la mejor posibilidad de su aporte, para acoger la regla general que señala que en materia de responsabilidad médica deben estar acreditados en el proceso todos los elementos que la configuran, para lo cual se puede echar mano de todos los medios probatorios legalmente aceptados, cobrando particular importancia la prueba indiciaria que pueda constituirse con fundamento en las demás pruebas que obren en el proceso, en especial para la demostración del nexo causal entre la actividad médica y el daño. En cuanto a la prueba del vínculo causal, de manera reciente se precisó la necesidad de demostrar en vinculo causal entre el daño y la actuación médica, que haga posible imputar a la entidad que prestó el servicio, el daño por el cual se demanda indemnización, el cual puede ser acreditado de manera indirecta mediante indicios (Consejo de Estado de Colombia, 2008a). 
Al respecto, se debe resaltar sobre la prueba del nexo causal que se puede demostrar mediante indicios, es decir, podemos afirmar que la probabilidad preponderante se sigue aplicando a los procesos de responsabilidad médica. El régimen adoptado recientemente, está encaminado a la acreditación del daño antijurídico en cabeza del demandante, acompañado de una imputación fáctica y jurídica respecto de la entidad que presuntamente causó el daño.

Desde luego, el Consejo de Estado es consciente de la dificultad que en ocasiones existe respecto de la demostración de alguno de los elementos configurativos de la responsabilidad por parte del demandante, debido a la imposibilidad de acceso a la información o por la falta de conocimiento respecto de alguna terapéutica caracterizada por su complejidad (Consejo de Estado de Colombia, 2011b). Por tal razón, se da la posibilidad de acreditar los elementos de forma indiciaria.

Respecto al nexo de causalidad el Consejo de Estado, recordó que en ocasiones es complicado de demostrar por la parte demandante, debido a su complejidad científica o técnica. Adicionalmente se arguyó que "por lo regular no queda huella de esa prestación, diferente al registro que el médico o el personal paramédico consigne en la historia clínica, la que además, permanece bajo el control de la misma entidad que prestó el servicio" (Consejo de Estado de Colombia, 2011a). En consideración con lo anterior, este Tribunal ha venido aplicando las teorías como res ipsa loquitur o la teoría de la causa adecuada, probabilidad preponderante.

Se trata de una situación que deja abierta la posibilidad de demostrar la relación de causalidad por la parte demandante, de manera amplia, de tal forma que existe un aligeramiento de la prueba de la parte demandante. Podemos afirmar, que, por regla general, el sistema aplicado actualmente es el de falla probada, y para la acreditación de los elementos constitutivos de responsabilidad está permitida la prueba indiciaria en materia del nexo de causalidad. 


\section{Conclusiones}

Desde hace algo más de 10 años el Consejo de Estado se decantó finalmente por establecer como título de imputación la falla probada. Esto indica que a cargo del demandante está la carga de probar el daño, la falla del servicio y su nexo de causalidad.

Para llegar al punto que el Consejo de Estado se encuentra se pasó por un ciclo, el cual partió y llego al mismo lugar, esto es la falla probada. En el intervalo de casi 16 años (1990-2006) el Consejo de Estado aceptó la falla presunta. Sin embargo, el Consejo de Estado se percató de la dificultad en los temas probatorios por parte de las instituciones públicas sanitarias.

En primer lugar, por el trascurso del tiempo entre el presunto daño y la presentación de la demanda. En segundo lugar, las empresas sociales del Estado manejan demasiados casos. Estas dos situaciones, según el máximo tribunal contencioso colombiano, hacen que en materia probatoria la Administración se encuentre en desventaja. De esta forma la carga de la prueba está a cargo del paciente. Pero sabiendo la dificultad de algunos tratamientos se dejó la posibilidad que el nexo de causalidad sea demostrado al menos de manera indiciaria.

Consideramos que este régimen de responsabilidad subjetiva es adecuado y se dan herramientas tanto a la parte demandante como a la demandada que le permiten estar en igualdad de condiciones desde el punto de vista probatorio.

\section{Referencias}

Consejo de Estado de Colombia. Sentencia de julio 7 de 1989, exp 5446 (1989).

Consejo de Estado de Colombia. Sentencia de octubre 24 de 1990, exp 5902 (1990).

Consejo de Estado de Colombia. Sentencia de agosto 24 de 1992, exp 6754 (1992).

Consejo de Estado de Colombia. Sentencia de julio 30 de 1992, exp 6897 (1992).

Consejo de Estado de Colombia. Sentencia del 13 de agosto de 1992, exp 7274 (1992). 
Consejo de Estado de Colombia. Sentencia de febrero 10 de 2000 exp 11878 (2000).

Consejo de Estado de Colombia. Sentencia agosto 31 de 2006, exp 15772 (2006).

Consejo de Estado de Colombia. Sentencia de agosto 31 de 2006, exp 15772 (2006).

Consejo de Estado de Colombia. Auto del 22 de mayo de 2008 (2008).

Consejo de Estado de Colombia. Sentencia agosto 26 de 2008, exp 15725 (2008).

Consejo de Estado de Colombia. Sentencia octubre 15 de 2008, exp 16270 (2008).

Consejo de Estado de Colombia. Sentencia abril 28 de 2010, exp 17725 (2010).

Consejo de Estado de Colombia. Sentencia de abril 28 de 2010, exp 20087A (2010).

Consejo de Estado de Colombia. Sentencia de abril 27 de 2011, exp 20502 (2011).

Consejo de Estado de Colombia. Sentencia del 7 de febrero de 2011, exp. 34387 (2011).

Consejo de Estado de Colombia. Sentencia de 25 abril de 2012, exp 21861 (2012).

Consejo de Estado de Colombia. Sentencia de abril 25de 2012, exp 21861 (2012).

Consejo de Estado de Colombia. Sentencia de julio 26 de 2012, exp 20732 (2012).

Consejo de Estado de Colombia. Sentencia 22 de octubre de 2012, exp 24776 (2012).

Consejo de Estado de Colombia. Sentencia 22 de octubre de 2012, exp 25242 (2012).

Consejo de Estado de Colombia. Sentencia de 7 de noviembre de 2012, exp 24525 (2012).

Consejo de Estado de Colombia. Sentencia 22 de noviembre de 2012, exp 26315 (2012). 
Consejo de Estado de Colombia. Sentencia de noviembre 22 de 2012, exp 25998 (2012).

Consejo de Estado de Colombia. Sentencia 3 de enero de 2013, exp 26999 (2013).

Consejo de Estado de Colombia. Sentencia 13 de febrero de 2013, exp 27253 (2013).

Consejo de Estado de Colombia. Sentencia 27 de febrero de 2013, exp 26009 (2013).

Consejo de Estado de Colombia. Sentencia 28 de febrero de 2013, exp 24460 (2013).

Consejo de Estado de Colombia. Sentencia 28 de febrero de 2013, exp 25075 (2013).

Consejo de Estado de Colombia. Sentencia 6 de marzo de 2013, exp 27132 (2013).

Consejo de Estado de Colombia. Sentencia 24 de abril de 2013, exp 27180 (2013).

Consejo de Estado de Colombia. Sentencia 3 de mayo de 2013, exp 22165 (2013).

Consejo de Estado de Colombia. Sentencia 3 de mayo de 2013, exp 26352 (2013).

Consejo de Estado de Colombia. Sentencia 31 de mayo de 2013, exp31724 (2013).

Consejo de Estado de Colombia. Sentencia 13 de junio de 2013, exp 28044 (2013).

Consejo de Estado de Colombia. Sentencia 24 de julio de 2013, exp 30309 (2013).

Consejo de Estado de Colombia. Sentencia 29 de agosto de 2013, exp 30283 (2013).

Consejo de Estado de Colombia. Sentencia 29 de agosto de 2013, exp 36465 (2013).

Consejo de Estado de Colombia. Sentencia 27 de septiembre de 2013, exp 24096 (2013).

Consejo de Estado de Colombia. Sentencia 30 de octubre de 2013, exp 24958 (2013). 
Consejo de Estado de Colombia. Sentencia 6 de diciembre de 2013, exp 28136 (2013).

Consejo de Estado de Colombia. Sentencia 12 de diciembre de 2013, exp 27493 (2013).

Consejo de Estado de Colombia. Sentencia 22 de enero de 2014, exp 28816 (2014).

Consejo de Estado de Colombia. Sentencia 12 de febrero de 2014, exp 27488 (2014).

Consejo de Estado de Colombia. Sentencia 27 de marzo de 2014, exp 26660 (2014).

Consejo de Estado de Colombia. Sentencia 27 de marzo de 2014, exp 35420 (2014).

Consejo de Estado de Colombia. Sentencia 14 de mayo de 2014, exp 33981 (2014).

Consejo de Estado de Colombia. Sentencia 14 de mayo de 2014, exp 30724 (2014).

Consejo de Estado de Colombia. Sentencia 23 de julio de 2014, exp 13260 (2014).

Consejo de Estado de Colombia. Sentencia 31 de julio de 2014, exp 27770 (2014).

Consejo de Estado de Colombia. Sentencia 12 de agosto de 2014, exp 29131 (2014).

Consejo de Estado de Colombia. Sentencia 10 de septiembre de 2014, exp 27365 (2014).

Consejo de Estado de Colombia. Sentencia 20 de octubre de 2014, exp 30166 (2014).

Consejo de Estado de Colombia. Sentencia 12 de noviembre de 2014, exp 30812 (2014).

Consejo de Estado de Colombia. Sentencia de noviembre 13 de 2014, exp 31182 (2014).

Consejo de Estado de Colombia. Sentencia de enero 28 de 2015, exp 30628 (2015).

Consejo de Estado de Colombia. Sentencia de marzo 5 de 2015, exp 30945 (2015). 
Consejo de Estado de Colombia. Sentencia de mayo 28 de 2015, exp 46466 (2015).

Consejo de Estado de Colombia. Sentencia de septiembre 29 de 2015, exp 21774 (2015).

Consejo de Estado de Colombia. Sentencia de mayo 31 de 2016, exp 33650 (2016).

Consejo de Estado de Colombia. Sentencia de enero 25 de 2017, exp 36816 (2017).

Corte Constitucional de Colombia. C-836 de agosto 9 de 2001 (2001).

Fernández Muñoz, M. F. (2008). La responsabilidad médica: problemas actuales. Bogotá: Temis.

Gil Botero, E. (2011). Responsabilidad extracontractual del Estado (5th ed.). Bogotá: Temis.

Gil Botero, E. (2012). La responsabilidad médica derivada de los actos administrativos en el sector salud. Bogotá: Universidad Externado de Colombia.

López Medina, D. E. (2009). El derecho de los juees (2nd ed.). Bogotá: Legis.

Parra Guzmán, M. F. (2010). Carga de la prueba en la responsabilidad médica. Bogotá: Doctrina y Ley.

Ruíz Orejuela, W. (2006). Responsabilidad del estado y sus regímenes. Bogotá: Ecoe.

Sarmiento García, M. G. (2001). La teoría objetiva de la responsabilidad en el artículo 90 de la Constitución. Revista de Derecho Privado, 47.

Serrano Escobar, L. G. (2007). Aspectos críticos de la responsabilidad médica en la actualidad. Bogotá: Doctrina y Ley.

Tamayo Jaramillo, J. (2001). Sobre la culpa de la prueba médica. Medellín: Dike. 
